\title{
Molecular Specification of Homopolymer of Vinylbenzyl-Lactose-Amide in Aqueous Solution
}

\author{
Isao Wataoka, Hiroshi Urakawa, Kazukiyo Kobayashi,* Kohji Ohno,** \\ Takeshi FuKuda, ${ }^{* *}$ Toshihiro AKaIKe,*** \\ and Kanji KaJIWARA ${ }^{\dagger}$ \\ Faculty of Engineering and Design, Kyoto Institute of Technology, \\ Matsugasaki, Sakyo-ku, Kyoto 606-8585, Japan \\ * Graduate School of Engineering, Nagoya University, \\ Chikusa-ku, Nagoya 464-8603, Japan \\ ** Institute for Chemical Research, Kyoto University, \\ Uji, Kyoto 611-0011, Japan \\ *** Faculty of Bioscience and Biotechnology, Tokyo Institute of Technology, \\ Midori-ku, Yokohama 227-8501, Japan
}

(Received November 30, 1998)

\begin{abstract}
The lactose-carrying polystyrene derivative, PVLA, is known as the material suitable for the incubation of liver cells and for drug delivery systems. PVLA was dissolved in $0.1 \mathrm{M}$ urea aqueous solution, and its structure was analyzed from the results of small-angle X-ray scattering and the molecular modeling. PVLA was found to have the shape of a molecular bottlebrush in solution, composed of a large pseudo helix of polystyrene backbone. The amphiphilic nature of the backbone and side chains is thought to determine the backbone conformation.

KEY WORDS Small-Angle X-Ray Scattering / Polymacromonomer / Glycopolymer / Lactose /
\end{abstract}

Recent advance in the precise polymerization technique has resulted in synthesizing many novel functional polymers, most of which mimic biopolymers. Those novel polymers are often found to be physiologically more active than the corresponding biopolymers. Thus in future, those novel materials are expected to compose a major biomedical system including cell separation, cell culture, drug delivery agent, and artificial antigen. Hybrids of synthetic polymers and biopolymers are of a particular interest, since the hybrid may enhance the characteristics of parent polymers. Carbohydrate is one of the most important candidates as a ligand or recognition signal, and a series of glycoconjugate polystyrene derivatives have been synthesized with varying the types of a pendant oligosaccharides. Here a convenient synthetic route ${ }^{1}$ was developed to construct the amphiphilic structure by arranging hydrophobic polystyrene main chains and hydrophilic pendant oligosaccharides. Synthesized glycoconjugate polymers were in fact found to function as a highly sensitive ligand. Highly concentrated multiantennary glyco signals along the hydrophobic main chains enhance the interaction with various types of carbohydrate-binding proteins. The enhancement is also attributed to the presence of the hydrophobic phenyl groups, although its active role is not clearly understood. ${ }^{2}$

The glycoconjugate polystyrene derivative is a type of comb-shaped polymers termed as polymacromonomers, where relatively long branches pendant from the backbone chain with a regular interval. An extensive research effort has been focused to prepare densely grafted polymacromonomers by radical polymerization. Although the monomer is a high molecular weight precursor composed of almost monodisperse polymers

\footnotetext{
$\dagger$ To whom correspondence should be addressed.
}

(or oligomers) with a vinyl end group, the radical polymerization results in polydisperse polymacromonomers. The living radical polymerization is an alternative route to prepare polymacromonomers with less polydispersity, but the molecular weight cannot be expected so high as in the conventional radical polymerization.

Small-angle X-ray and light scattering revealed a bottlebrush-like cylindrical shape representing the polymacromonomer composed of a polymethacrylate backbone with oligostyrene side chains in toluene solution. ${ }^{3,4}$ The polymethacrylate backbone seems to be obliged to take an extended conformation in order to accommodate overcrowded side chains. In fact, the main chain seems to assume an extended almost zigzag conformation, and in consequence mesophase formation was observed from highly concentrated solutions of polymacromonomer. ${ }^{5}$ At lower degrees of polymerization, both the backbone and side chains seem to assume almost the same conformation as the linear homologues, so that the conformational change of the backbone chain should take place when the degree of polymerization exceeds a certain limiting value. ${ }^{6}$

In an earlier work, we have reported the structure of glycopolymer composed of a polystyrene backbone with pentamaltose side chains in aqueous solution. ${ }^{7}$ Here the small-angle X-ray scattering indicated the glycopolymer as represented by a cylinder with one or two broken points, and its backbone was found to assume a large cross-sectional pseudo-helix. Since the side chains are not long enough to fill the space along the extended hydrophobic backbone, the backbone is thought to assume a large helical conformation in order to cover its surface with hydrophilic side chains. A similar method is applied for the structural characterization of lactose-carrying polystyrene in aqueous solution in the present work. Lactose-carrying polystyrene is known as 
the material suitable for the incubation of liver cells and the drug delivery systems. ${ }^{8}$ The physiological activity was found to depend on the type of the backbones, which determine the side chain conformation. That is, the side chain conformation with respect to the backbone is considered to play a key role in the physiological activity of glycoconjugate polymers. In this context, the present work focuses the conformational analysis of glycoconjugate polymers by combining the small-angle $\mathrm{X}$-ray scattering and the molecular simulation.

\section{EXPERIMENTAL}

\section{Lactose-Carrying Polystyrene}

Three lactose-carrying polystyrene samples are employed for the present work. PVLA was obtained by radical homopolymerization of the monomer with the initiator, so that the molecular weight distribution would be rather broad. ${ }^{1}$ The other two samples VLA29 and VLA92 were prepared by a living radical polymerization, ${ }^{9}$ and the degree of polymerization were estimated as 29 and 92, respectively, by GPC. The molecular weight distribution $M_{w} / M_{n}$ of VLA29 and VLA92 are found to be 1.12 and 1.22 , respectively. The synthesis details will be found in the separate papers. ${ }^{1,9}$

\section{Small-Angle X-Ray Scattering}

Small-angle X-ray scattering (SAXS) was observed from the $0.1 \mathrm{M}$ urea solutions of lactose-carrying polystyrenes. The measurement was performed with a SAXES $^{10}$ (small-angle X-ray scattering equipment for solutions) installed at the BL10C of Photon Factory, Tsukuba, Japan. An incident X-ray from synchrotron radiation was monochromatized to $\lambda=1.488 \AA$, and then focused to the detector position. The scattered X-ray was detected by the one-dimensional position sensitive proportional counter (PSPC) of the effective length $160 \mathrm{~mm}$. The exact camera length was calibrated by using the diffraction peaks of collagen fiber (the long period $=653 \AA$ at the 6 th, 9th, and 11 th orders). The SAXS intensities were accumulated to the total measuring time of $30 \mathrm{~min}$. The sample solutions were prepared by weighing lactose-carrying polystyrene directly in the sample bottle and adding solvent to adjust the concentration of the solutions. The solutions were put into the flat cells $(1 \mathrm{~cm} \times 0.5 \mathrm{~cm} \times 0.1 \mathrm{~cm})$ equipped with a pair of thin quartz glass windows, which were subjected to the SAXS measurements. The cells were thermostated at $25^{\circ} \mathrm{C}$ by circulating water of a constant temperature through the cell holder. The scattered intensities were corrected with respect to the variation of the incident X-ray flux by monitoring the beam with an ionizing chamber placed in front of the thermostated sample holder. The excess scattering intensities were evaluated by subtracting the scattering intensities of solvent from those of lactose-carrying polystyrene solutions. $0.1 \mathrm{M}$ urea was added to the aqueous solutions of lactose-carrying polystyrene in order to prevent aggregation by intermolecular hydrogen bonding.

\section{Molecular Model}

The molecular model was generated by the use of the program Cerius2 ver 3.5 (BIOSYM/Molecular Simula-

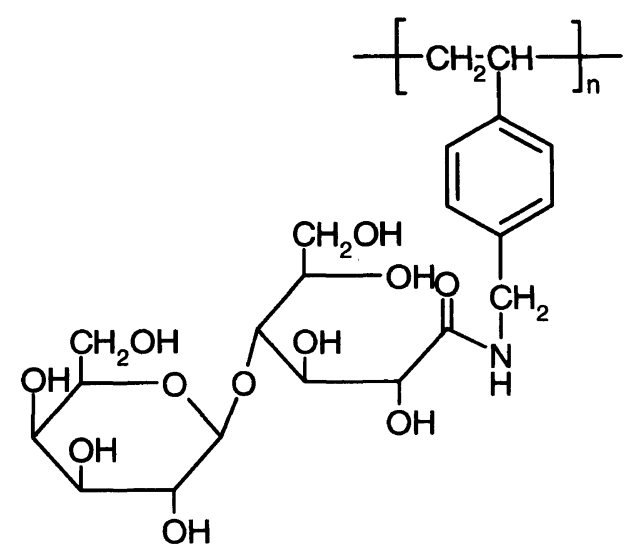

Figure 1. Chemical structure of PVLA.

tions) installed in the Silicon Graphics $\mathrm{O}_{2}$ series workstation. The universal force field ${ }^{11} 1.02$ was used for energy minimization and molecular dynamics. The molecular mechanics minimization was applied at first to the molecular model constructed manually. The resulted model from the molecular mechanics minimization was annealed by affording a thermal disturbance, and then the energy-minimization was performed in the system containing solvent by the use of a conjugate gradient $^{12}$ algorithm, until the root mean square force was less than $0.10 \mathrm{kcal} \mathrm{mol}^{-1}$ or the number of the minimization step exceeded 3000 in the case of the initial minimization. The molecular dynamics simulation for annealing was conducted at a constant volume under the conditions identical with those described for the energy minimization by the molecular mechanics. The temperature of the system was initially fixed at $300 \mathrm{~K}$, increased to $500 \mathrm{~K}$ and then cooled to $300 \mathrm{~K}$ with the temperature increment or decrement of $50 \mathrm{~K}$ per 50 steps. One cycle consists of 50 steps at each temperature from $300 \mathrm{~K}$ to $500 \mathrm{~K}$ and then down to $350 \mathrm{~K}$, and the elapsed time for 1 step corresponds to $0.0010 \mathrm{ps}$. Thus the total simulation time amounts $2.05 \mathrm{ps}$.

The particle scattering factor is calculated for lactose-carrying polystyrene from the atomic coordinates of the simulated polymacromonomer molecule according to the Debye formula. ${ }^{13}$ The scattering profile is given by

$$
I(q)=\sum_{i=1}^{n} g_{i}^{2} \phi_{i}^{2}(q)+2 \sum_{i=1}^{n-1} \sum_{j=i+1}^{n} g_{i} g_{j} \phi_{i}(q) \phi_{j}(q) \frac{\sin d_{i j} q}{d_{i j} q}
$$

where $q$ is the magnitude of scattering vector given by $(4 \pi / \lambda) \sin \theta$ with $\theta$ and $\lambda$ being a half of the scattering angle and the wavelength of an incident X-ray, respectively. $g_{i}$ is an atomic scattering factor and $d_{i j}$ is the distance between $i$ th and $j$ th atoms. The form factor for the $i$ th atom $\phi_{i}(q)$ is assumed to be given by the form factor of a sphere having a radius equivalent to a van der Waals radius of the $i$ th atom as

$$
\phi_{i}(q)=\frac{3\left[\sin \left(R_{i} q\right)-\left(R_{i} q\right) \cos \left(R_{i} q\right)\right]}{\left(R_{i} q\right)^{3}}
$$

with $R_{i}$ being the van der Waals radius of the $i$ th atom. 


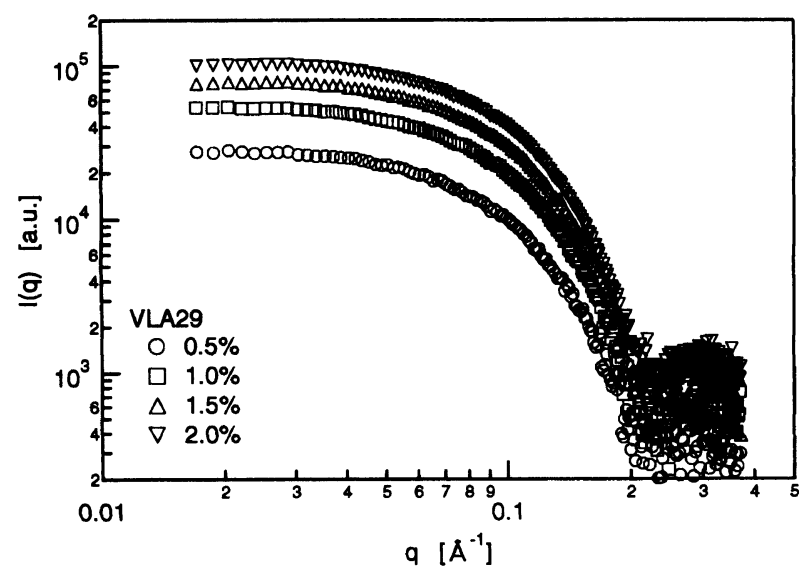

Figure 2. Double logarism plot for the glycopolymer solution with several concentration of VLA29 in $0.1 \mathrm{M}$ urea aqueous solutions in SAXS.

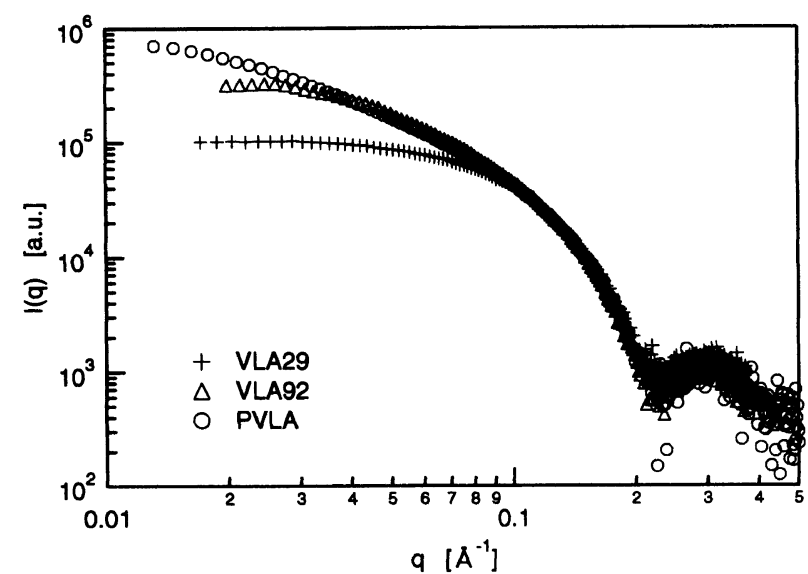

Figure 3. SAXS profile of VLA29, VLA92, and PVLA. The concentrations of each solution are the same $(2 \mathrm{wt} \%)$.

\section{RESULTS AND DISCUSSION}

Figure 2 shows the SAXS profiles from PVLA29 in $0.1 \mathrm{M}$ urea aqueous solution. The SAXS profile reveals a characteristic minimum at about $q=0.23 \AA^{-1}$. No concentration effect was observed in the scattering profiles, as the normalization with respect to the concentration yielded almost an identical profile. Figure 3 shows the SAXS profiles for different degrees of polymerization of these glycopolymers. The molecular weight dependence is observed only in low $q$ region. At $q>0.1 \AA^{-1}$, these profiles coincide, indicating that the conformation of glycopolymers is almost the same regardless of the molecular weight and the SAXS difference at low $q$ region is due to the size of whole molecule. All the profiles have the minimum of the scattering intensities at $q=0.23 \AA^{-1}$. Since the pentamaltose-carrying polystyrene has shown the scattering minimum at $q=0.18 \AA^{-1}$, the position of the scattering minimum seems to be characteristic of the type and length of oligosaccharide side chains.

The scattering intensity $I(q)$ at smaller scattering angles is approximately given according to Guinier ${ }^{14}$ as:

$$
I(q) \sim \exp \left(\frac{-q^{2} R_{\mathrm{g}}^{2}}{3}\right)
$$

Table I. Radius of gyration and cross-sectional radius of gyration evaluated from SAXS or calculated from the molecular model

\begin{tabular}{lcccccc}
\hline \multirow{2}{*}{ Sample } & \multicolumn{2}{c}{$R_{\mathrm{g}} / \AA$} & & \multicolumn{2}{c}{$R_{\mathrm{gc}} / \AA$} \\
\cline { 2 - 3 } \cline { 5 - 6 } & Obsd & Calcd & & Obsd & Cald \\
\hline VLA29 & 17.9 & 17.5 & & - & - \\
VLA92 & - & - & & 13.1 & 14.5 \\
PVLA & - & - & & 12.5 & - \\
\hline
\end{tabular}

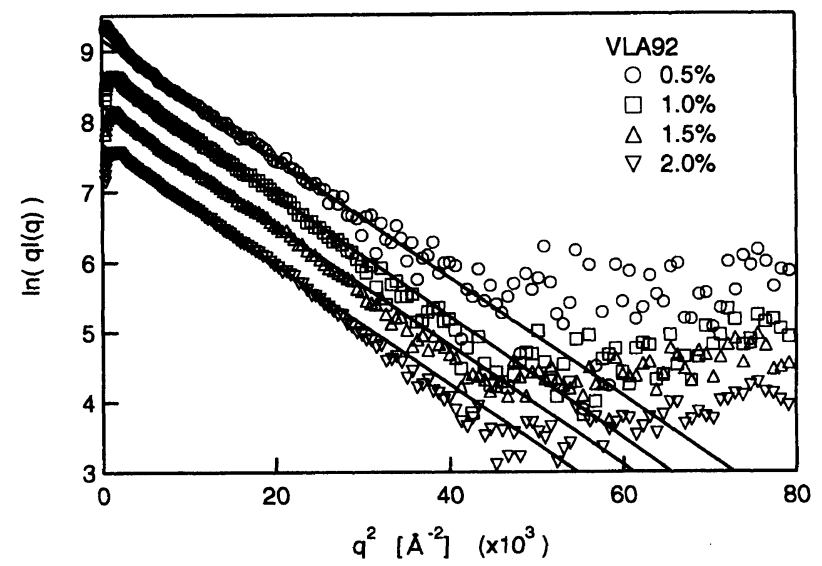

Figure 4. Cross-sectional Guinier plots for VLA92 in four concentrations. Full lines show Guinier approximation.

with a single parameter $R_{\mathrm{g}}$ defined as the radius of gyration of a solute molecule. Eq 3 implies that the radius of gyration of the molecule is determined from the initial slope of $I(q)$ plotted against $q^{2}$ (referred to as the Guinier plots). The radius of gyration can be evaluated from the linear region of Guinier plots when the condition $q^{2} R_{\mathrm{g}}^{2} \leq 1$ is satisfied. The condition was satisfied for VLA29, and the radius of gyration was estimated as $R_{\mathrm{g}}=17.9 \AA$ (see also Table I). The condition was not satisfied for VLA92 and PVLA, which were too large $\left(q^{2} R_{\mathrm{g}}^{2} \gg 1\right)$ in a whole range of the observed $q$.

When a molecule is rod-like, the scattering from such a molecule is approximately described in terms of the cross-sectional radius of gyration $R_{\mathrm{gc}}$ as ${ }^{15}$

$$
q I(q) \sim \exp \left(\frac{-q^{2} R_{\mathrm{gc}}^{2}}{2}\right)
$$

The relationship holds in the range where $q R_{\mathrm{g}} \geq 1$ and $q R_{\mathrm{gc}} \leq 1$, and $R_{\mathrm{gc}}$ is determined from the slope of $\ln q I(q)$ plotted against $q^{2}$ (referred to as the cross-sectional Guinier plots) in the appropriate range of $q$. The example of the cross-sectional Guinier plots is shown for VLA92 in Figure 4, and the values of $R_{\mathrm{gc}}$ were evaluated as summarized in Table I with the $R_{\mathrm{g}}$ values of VLA29. The conformation of the main chain in VLA92 and PVLA is almost identical as suggested by the $R_{\mathrm{gc}}$ values confirming a similar cross-sectional size.

The lactose-carrying polystyrene was also found to be represented by a cylinder with a large cross-sectional radius of gyration. The cross-sectional radius of gyration of PVLA was estimated as $12.5 \AA$, which is accounted for by considering a pseudo large helix. Although pentamaltose-carrying polystyrene assumes a similar backbone conformation, ${ }^{8}$ a smaller cross-sectional radius 


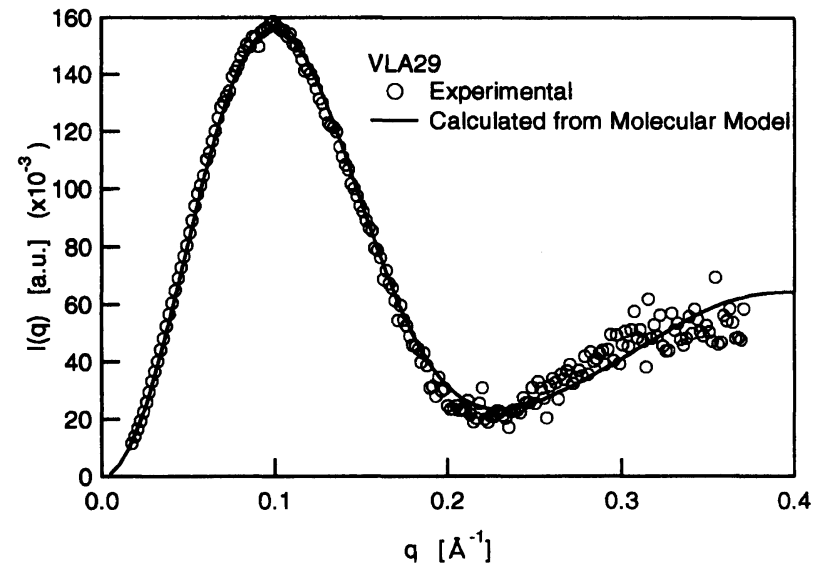

Figure 5. Kratky plots of the experimental data and the one calculated from the molecular model shown in Figure 6. Circles denote the observed SAXS intensities and full line indicates the calculated scattering profile.

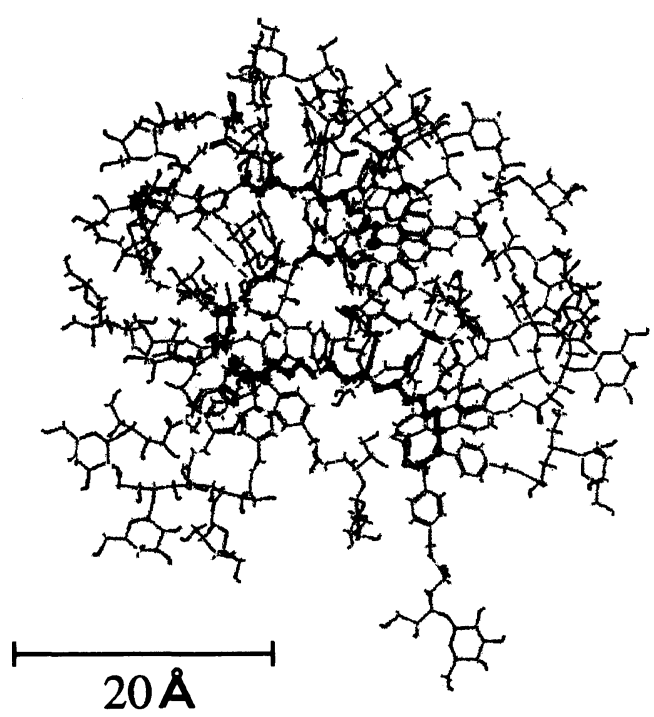

Figure 6. The molecular model of VLA29. The main chain is represented by a ball and stick model.

was estimated for pentamaltose-carrying polystyrene than lactose-carrying polystyrene. A shorter side chain probably requires a larger cross-sectional radius to reduce the hydrophobic surface to cover.

In order to account for a cylindrical shape with a large cross-section, these polymers are supposed to be composed of a polystyrene spiral with protruding lactose side chains. Based on this conjecture, the molecular models of VLA29 were constructed by Cerius2, and the particle scattering factor calculated from each generated chain was compared with the observed SAXS profile. Figure 6 shows a molecular model of VLA29, which yields the radius of gyration $R_{\mathrm{g}}=17.5 \AA$ and exhibits a scattering profile similar to the observed SAXS profile (see Figure 5). The molecular model was found to be represented by an ellipsoid rather than a cylinder. Since the degree of polymerization is low, the effect of hydrophobic chain ends cannot be neglected and in consequence the conformation toward the backbone chain ends is disturbed. A similar tendency was observed in the toluene solutions of polymacromonomers of lower degrees of polymerization. ${ }^{7}$ The increase of the degree

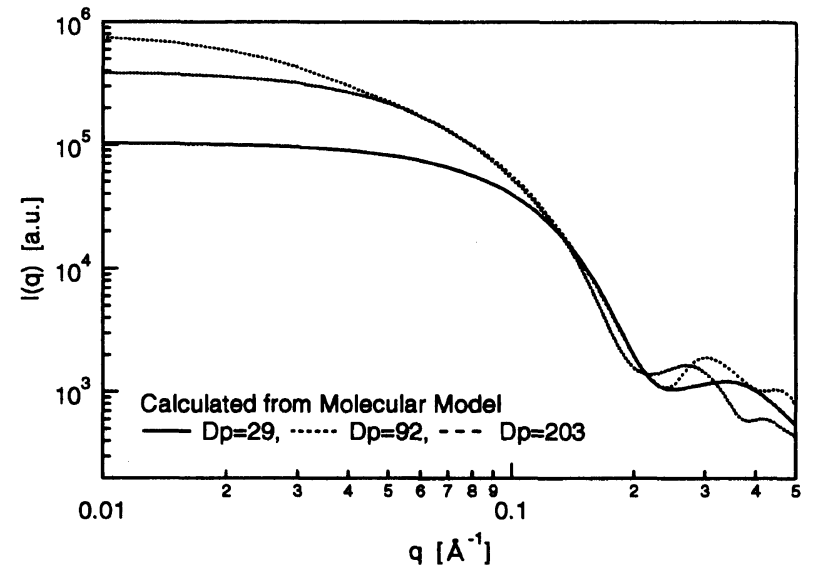

Figure 7. SAXS profiles for difference degrees of polymerization of the molecular models in the double logalism plots. The full line, the dotted line, and the broken line indicate the molecular model for $D P=29, D P=87$, and $D P=203$, respectively.

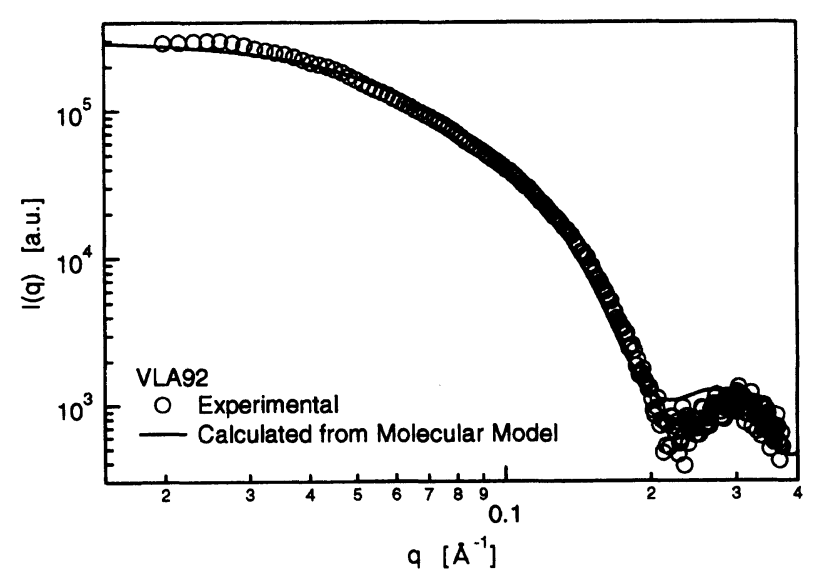

Figure 8. Double logalism plots of the experimental data and the one calculated from the molecular model shown in Figure 9. Circles denote the observed SAXS intensities and full line indicates the calculated scattering profile.

of polymerization in a molecular model results in the change of the scattering profile mainly in the small $q$ region (Figure 7). However, the overall shape becomes more marked as a cylinder than an ellipsoid as the degree of polymerization increases. Figure 8 shows the SAXS profile calculated from a molecular model of VLA92 $\left(R_{\mathrm{gc}}=14.5 \AA\right)$ (Figure 9). The backbone chain assumes a pseudo-helical conformation for lactose side chains to cover the surface of polystyrene backbone. Since no stereo-regularity was expected from the polymerization scheme, the backbone chain conformation should be fundamentally random in terms of the sequence of trans and gauche. The observed scattering profiles show a consistent increase of the intensity in accordance with the increase of the degree of polymerization in the region of $q<0.1 \AA^{-1}$. The scattering profiles are almost identical at $q>0.1 \AA^{-1}$ regardless of the degree of polymerization. The polymacromonomer composed of a polymethylmethacryate backbone with polystyrene side chain showed a conformational change of the backbone at about $D P_{w}=20-30$ when the degree of polymerization was increased. Both the backbone and side chains follow approximately Gaussian statistics in the polymacromonomer of lower degrees of polymerization. The 


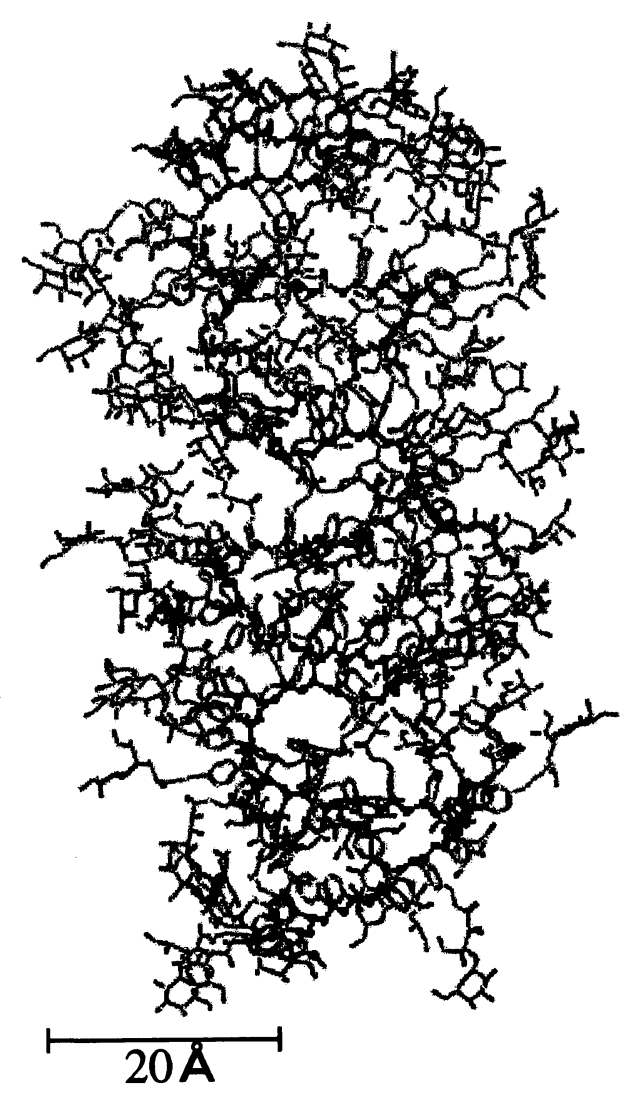

Figure 9. The molecular model of VLA92. The main chain is represented by a ball and stick model.

excluded volume of overcrowded side chains takes into effect and forces the backbone assuming more extended (almost zigzag) conformation in the polymacromonomer of higher degrees polymerization.

Lactose-carrying polystyrene retains its pseudo-helical conformation in water even at $D P_{w}=29$. In this case, the backbone conformation is determined not by the excluded volume effect of side chains, but by the amphiphilic character of the backbone (hydrophobic) and side chains (hydrophilic). The amphiphilicity obliges for lactose-carrying polystyrene to form a cylindrical micelle in aqueous solution. In this context, the lactose-carrying polystyrene may be regarded as a molecular micelle.

\section{CONCLUSION}

Lactose-carrying polystyrene was characterized as the shape of a molecular bottlebrush in the $0.1 \mathrm{M}$ urea aquarius solution. Its backbone chain is made of the pseudo-helix with a relatively large cross-section. The cross-section of the backbone was found to be larger than that of maltopentaose-carrying polystyrene. Lactose-carrying polystyrene with very low degree of polymerization, $D P_{w}=29$, maintains the pseudo-helical conformation as the polymer of high molecular weights. The backbone conformation seems to be determined not by the excluded volume effect of side chains, but by the amphiphilic character of the backbone (hydrophobic) and side chains (hydrophilic). That is, the lactosecarrying polystyrene may be regarded as a molecular micelle.

Acknowledgment. This work was performed under the approval of the Photon Factory Advisory Committee (Proposal No. 94G293, 97G139). We acknowledge the financial support from the Grant-in-Aid for Scientific Research on Priority Areas from the Ministry of Education, Science, Sports and Culture of Japan.

\section{REFERENCES}

1. K. Kobayashi, H. Sumitomo, and Y. Ina, Polym. J., 17, 567 (1985).

2. K. Kobayashi, A. Tsuchida, T. Usui, and T. Akaike, Macromolecules, 30, 2016 (1997).

3. M. Wintermantel, M. Schmidt, Y. Tsukahara, K. Kajiwara, and S. Kohjiya, Macromol. Rapid Commum., 15, 279 (1994).

4. M. Wintermantel, M. Gerle, K. Fischer, M. Schmidt, I. Wataoka, H. Urakawa, K. Kajiwara, and Y. Tsukahara, Macromolecules, 29, 978 (1996).

5. M. Wintermantel, K. Fischer, M. Gerle, R. Ries, M. Schmidt, K. Kajiwara, H. Urakawa, and I. Wataoka, Angew. Chem. Int. Ed. Engl., 34, 1472 (1995).

6. I. Wataoka, H. Urakawa, K. Kajiwara, M. Schmidt, and M. Wintermantel, Polym. Int., 44, 365 (1997).

7. I. Wataoka, H. Urakawa, K. Kobayashi, T. Akaike, M. Schmidt, and K. Kajiwara, Macromolecules, in press.

8. M. Goto, H. Yura, C.-W. Chang, A. Kobayashi, T. Shinoda, A. Maeda, S. Kojima, K. Kobayashi, and T. Akaike, J. Controlled Release, 28, 223 (1993).

9. K. Ohno, Y. Tsujii, T. Miyamoto, T. Fukuda, T. Goto, K Kobayashi, and T. Akaike, Macromolecules, 31, 1064 (1998).

10. T. Ueki, Y. Hiragi, Y. Izumi, H. Tagawa, M. Kataoka, Y. Muroga, and Y. Amemiya, Photon Factory Activity Report, 1, V7, V29, V170 (1983).

11. A. K. Rappe, C. J. Casewit, K. S. Colwell, W. A. Goddard, and W. M. Skiff, J. Am. Chem. Soc., 114, 10024 (1992).

12. R. Fletcher and C. M. Reeves, Comput. J., 7, 149 (1964).

13. O. Glatter, Acta Physica Austriaca, 52, 243 (1980).

14. A. Guinier, Ann. Phys., 12, 161 (1939).

15. See, for example, O. Glatter, and O. Kratky, Ed., "Small Angle X-ray Scattering,” Academic Press, London, 1982. 